\title{
Variabilidade genética e alterações morfológicas em progênies de polinização aberta de Eucalyptus grandis sob o efeito de paclobutrazol
}

\section{Genetic diversity and morphological changes in open pollination progenies of Eucalyptus grandis under paclobutrazol regulator effects}

\author{
Cristiano Bueno de MORAES ${ }^{1,2}$; André CARIGNATO ${ }^{3}$; Gláucia UESUGI ${ }^{4}$; \\ Fernanda Maria ABÍLIO ${ }^{5}$; Edwin Camacho PALOMINO ${ }^{6}$; Edson Seizo MORI \\ ${ }^{1}$ Parte da dissertação de mestrado do primeiro autor; Pesquisa financiada pela Fundação de Amparo à Pesquisa \\ do Estado de São Paulo - FAPESP \\ ${ }^{2}$ Prof. Dr.; Universidade Federal do Tocantins - Unidade de Gurupi; cb_moraes2014@yahoo.com.br \\ ${ }^{3}$ Autor para correspondência; Graduando em Engenharia Florestal; Universidade Estadual Paulista "Júlio de \\ Mesquita Filho" - Faculdade de Ciências Agronômicas de Botucatu; Departamento de Produção e \\ Melhoramento Vegetal; Rua José Barbosa de Barros, 1780, Caixa Postal 237, CEP 18610-307 - Botucatu - \\ SP; andrecarignatoflorestal@hotmail.com \\ ${ }^{4}$ Doutoranda em Ciência Florestal; Universidade Estadual Paulista "Júlio de Mesquita Filho" - Faculdade de \\ Ciências Agronômicas de Botucatu; ci_uesugi@yahoo.com.br; \\ 5 Engenheira Florestal, UNESP/FCA; fernandama@eucatex.com.br \\ ${ }^{6}$ Prof. Dr.; Universidade Federal do Oeste do Pará; edwincamacho2@hotmail.com; \\ 7 Prof. Ph.D; Universidade Estadual Paulista "Júlio de Mesquita Filho" - Faculdade de Ciências Agronômicas de \\ Botucatu; esmori@fca.unesp.br
}

Recebido em: 25-03-2014; Aceito em: 05-08-2014

\section{Resumo}

O presente trabalho teve como objetivo estudar a variabilidade genética e as alterações das características morfológicas em progênies de polinização aberta de Eucalyptus grandis sob o efeito do biorregulador de crescimento paclobutrazol. O experimento foi instalado no delineamento em blocos casualizados. As modificações que ocorreram antes e durante a indução floral das plantas foram estudadas e os parâmetros genéticos estimados para altura de plantas e diâmetro do caule. O regulador paclobutrazol causou modificações morfológicas durante o desenvolvimento das plantas; que foram intensas logo após a aplicação do regulador e foram diminuindo seu efeito ao longo das avaliações. Os coeficientes de variação mostraram haver maior variabilidade genética dentro do que entre progênies, para as características estudadas, apontando para maior eficiência na seleção dentro das progênies em programas de melhoramento do Eucalyptus grandis.

Palavras-chave adicionais: eucalipto; parâmetros genéticos; regulador vegetal.

\begin{abstract}
The research objective was to study the genetic diversity of morphological traits in Eucalyptus grandis progenies growing under paclobutrazol regulator effects. The progeny trail was set up through design of randomized blocks. The morphological changes occurred before and during the plant flowering were analyzed. The estimation of genetic parameters were for plant height and stem diameter. The paclobutrazol have caused changes on plant development being strong by the beginning and becoming lightening through the evaluations. The coefficients of variation have shown there is higher genetic diversity within than among progenies for the studied traits. Therefore, it can have high efficiency on selection within progenies in the Eucalyptus grandis breeding program.
\end{abstract}

Additional keywords: eucalypt; genetic parameter; plant growth regulator.

Introdução

O eucalipto é o gênero florestal mais usado nos reflorestamentos no Brasil, em razão de suas características de rápido crescimento e boa adaptação às condições edafoclimáticas existentes no País. Dentre suas espécies existentes, o Eucalyptus grandis Hill ex Maiden, pertencente à família das Mirtaceas (SOARES et al., 2003; ALMEIDA et al., 2007), ocupa uma das maiores áreas plantadas no território brasileiro (SOUZA et al., 2004). 
Entretanto, muitas dificuldades ainda são encontradas na implantação de programas de melhoramento visando à obtenção de ganhos genéticos para características silviculturais para a espécie. O período de florescimento, a ocorrência da primeira florada, a intensidade na floração e a irregularidade de florescimento, são alguns dos problemas encontrados na condução de um programa de melhoramento (WILLIANS et al., 2003; JONES et al., 2011).

Esses problemas tornam-se ainda mais importantes à medida que estudos demonstram a efetividade da seleção em idades precoces, momento em que as plantas ainda não são capazes de se reproduzir (NETO et al., 2003; ZIMBACK et al., 2011). Desta forma, a duração das gerações de melhoramento poderia ser reduzida por métodos que induzam o aparecimento de flores em plantas juvenis (YUCEER et al., 2003).

O paclobutrazol [(2RS - 3RS $)-1-(4-$ clorofenil) - 4, 4, dimetil - 2 - (1H-1,2,4-triazol-1-il) pentan-3-ol], fórmula empírica $\mathrm{C}_{15} \mathrm{H}_{20} \mathrm{CIN}_{3} \mathrm{O}$, é um composto químico, derivado do triazol, que atua na planta como inibidor de crescimento (BERBERICH et al., 2006), auxiliando também na formação e na indução de gemas florais (GARDNER et al., 2013). Ele promove inibição da biossíntese de giberelina (MARSHALL et al., 2000; TAIZ \& ZEIGER, 2009), provocando modificações morfológicas em determinados gêneros e espécies. No caso de angiospermas lenhosas, essa inibição está relacionada à parada do crescimento vegetativo e o estímulo do estágio reprodutivo, tendo como consequência a iniciação precoce de botões florais.

As plantas sob o efeito do biorregulador de crescimento paclobutrazol sofrem muitas alterações morfológicas (MORAES et al., 2012; MORAES et al., 2013). CHORBADJIAN et al. (2011), em estudo com Pinus nigra e Betula papyrifera, observaram que 0 paclobutrazol reduziu a altura e o diâmetro do caule em ambas as espécies. $\mathrm{O}$ efeito dos reguladores de crescimento ocorre por bloqueio da ação ou biossíntese das giberelinas, e alguns dos principais efeitos observados ocorrem na elongação e na divisão de células nos meristemas lateral e apical.

Apesar de o paclobutrazol ser um biorregulador de crescimento muito utilizado para auxiliar na indução precoce da floração, em diferentes gêneros e espécies, incluindo o eucalipto, raros são os estudos mostrando as modificações morfológicas e a variabilidade genética em progênies de $E$. grandis sob seu efeito.

Portanto, a presente pesquisa teve como objetivo principal estudar a variabilidade genética e as alterações morfológicas em progênies de polinização aberta de Eucalyptus grandis sob o efeito do biorregulador de crescimento.

\section{Material e métodos}

O experimento foi conduzido no viveiro de produção de mudas florestais do Departamento de Recursos Naturais - Ciências Florestais, da Faculdade de Ciências Agronômicas, FCA/UNESP, Botucatu-SP. As progênies de Eucalyptus grandis utilizadas no experimento são de procedência de Coffs Harbour, originárias da Austrália e oriundas do programa de melhoramento da empresa Eucatex S/A.

$O$ experimento foi instalado em blocos casualizados: vinte progênies, seis plantas por parcela e três repetições (PIMENTEL-GOMES, 2009), sendo cinco plantas com aplicação de paclobutrazol e uma testemunha (sem paclobutrazol), totalizando 360 vasos. A aplicação foi feita aos seis meses de idade. $O$ produto paclobutrazol foi aplicado via solo, na dosagem de 1,0 $\mathrm{mL}$ do produto comercial CULTAR, diluído em $300 \mathrm{~mL}$ de água destilada, na concentração de $25 \%$ de ingrediente ativo.

As análises morfológicas, medições de altura e diâmetro do caule foram realizadas de dois em dois meses após a aplicação do biorregulador vegetal, sendo a primeira avaliação feita quando as mudas completaram 12 meses de idade, e a última aos 20 meses, totalizando cinco avaliações.

\section{Análise estatística}

Com base nos dados coletados de altura e diâmetro do caule, foram estimados os parâmetros genéticos quantitativos, utilizando o programa computacional GENES, que se baseia no modelo de STEEL \& TORRIE (1980), considerando-se todos os efeitos como aleatórios, excetuando-se a média (CRUZ, 2001).

A estrutura da análise de variância utilizada pelo programa computacional para a obtenção das estimativas das variâncias, no nível de média de parcelas, está exposta na Tabela 1, conforme este modelo matemático:

$Y i j=M+T i+B j+T B i j+E i j$

Na fórmula, Yij é a observação do genótipo i no bloco j;

$M$ é o efeito médio para o caráter;

Bj é o efeito do bloco;

Ti é o efeito do tratamento $\mathrm{i}$;

TBij é a interação entre tratamento i e o bloco j, e Eij é o efeito residual devido a cada parcela.

As análises estatísticas foram feitas através do software SAS for Windows (versão 9.1.3), comparando-se as médias entre progênies pelo teste de Tukey, a $5 \%$ de probabilidade (VIEIRA, 2006). 
Tabela 1 - Esquema da estrutura da análise de variância. Analysis of variance scheme.

\begin{tabular}{|c|c|c|c|c|}
\hline Fator de Variação & G.L. & SQ & QM & $E(Q M)$ \\
\hline Blocos (b) & $r-1$ & SQb & $\mathrm{SQb} /(r-1)$ & ------ \\
\hline Progênies (p) & $t-1$ & SQp & $\mathrm{SQp} /(\mathrm{t}-1)=$ & $(1 / n) \sigma_{d}^{2}+\sigma_{e}^{2}+r \sigma_{p}^{2}$ \\
\hline Erro (e) & $(r-1)(t-1)$ & SQe & $\mathrm{SQe} /(\mathrm{r}-1)(\mathrm{t}-1)=$ & $(1 / n) \sigma_{d}^{2}+\sigma_{e}^{2}$ \\
\hline TOTAL & $(\mathrm{rt})-1$ & SQ total & -------- & ------- \\
\hline
\end{tabular}

\section{Resultados e discussão}

\section{Alterações morfológicas decorrentes do uso do biorregulador paclobutrazol}

As alterações morfológicas apresentadas pelas plantas neste experimento foram semelhantes às encontradas por RIBEIRO et al. (2011), que constataram em Helianthus annuus a inibição do crescimento em altura, a redução do crescimento em geral dos indivíduos, o curvamento acentuado da nervura principal das folhas e entrenós. Segundo QIANSHENG et al. (2009), em experimentos com monguba (Pachira aquatica) e MORAES et al. (2013), com a espécie jatobá (Hymenaea courbari), a redução no crescimento de entrenós é um dos efeitos mais frequentes causados pelo biorregulador paclobutrazol. Também foi apresentada, neste trabaIho, quebra de dormência das gemas laterais, ao longo do caule, conforme pode ser observado pelo desenvolvimento das brotações nos indivíduos (Figura 1A).
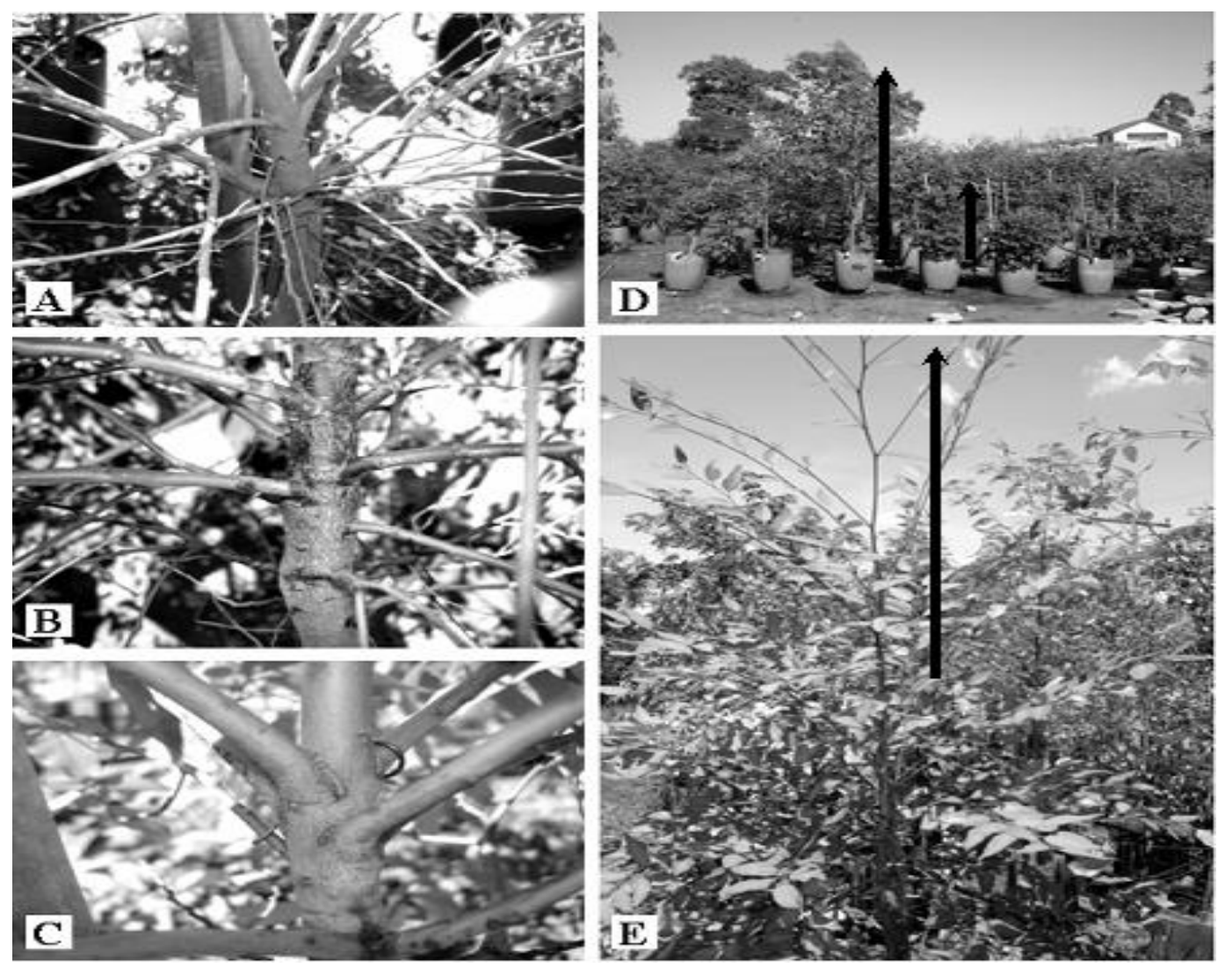

Figura 1 - Alterações morfológicas em Eucalyptus grandis decorrentes da aplicação de paclobutrazol: (A) brotações acentuadas no caule, (B) caule com aspecto envelhecido, (C) entrenós mais curtos, (D) redução do crescimento, seta maior (planta sem paclobutrazol), seta menor (planta com paclobutrazol) e (E) desenvolvimento apical. Morphological modifications in Eucalyptus grandis because of paclobutrazol application: (A) intense sprouting on stem, (B), stem with mature aspects, (C) shorter internodes, (D) reduction of growth, bigger arrow (plant without paclobutrazol), shorter arrow (plant with paclobutrazol), and (E) apical development. 
Outro sintoma que as plantas submetidas ao tratamento com o biorregulador paclobutrazol apresentaram, foi o ressecamento das folhas terminais, e os limbos das folhas maduras tornaram-se mais coriáceos. No caule, observou-se, também, um aspecto semelhante ao indivíduo maduro (adulto), que se apresentou com aspecto envelhecido (Figura 1B), aspectos também observados em estudo com $E$. grandis por MORAES et al. (2012).

GRIFFIN et al. (1993), aplicando paclobutrazol em Eucalyptus nitens, com dois anos de idade, constataram redução no crescimento em altura das plantas, após 6 meses de tratamento. HASAN \& REID (1995) também constataram redução na altura total de mudas de Eucalyptus globulus no tratamento com paclobutrazol. Estes resultados são semelhantes aos encontrados neste estudo (Figura 1D).

Dentre as progênies submetidas ao tratamento com paclobutrazol, a que apresentou efeito mais acentuado na redução em altura foi a progênie 15 (Tabela 2), mostrando diferenças significativas na primeira e segunda avaliações. No entanto, a partir da $3^{\mathrm{a}}$ avaliação, em geral, foi observado que os indivíduos retomaram seu crescimento meristemático apical, devido possivelmente à diminuição do efeito do produto paclobutrazol sobre os indivíduos das famílias (Figura 1E).

Tabela 2 - Valores médios de altura das plantas das progênies de Eucalyptus grandis aos 12; 14; 16; 18 e 20 meses (avaliações 1, 2, 3, 4 e 5, respectivamente) sob efeito da aplicação de paclobutrazol e testemunha (sem aplicação). Averages of plants progeny heights of Eucalyptus grandis by 12, 14, 16, 18 and 20 months (1, 2, 3, 4, and 5 evaluations, respectively) under paclobutrazol effects and control (no application).

\begin{tabular}{|c|c|c|c|c|c|c|c|c|c|c|c|}
\hline \multirow[t]{2}{*}{${ }^{1} \mathrm{P}$} & \multicolumn{3}{|c|}{$\begin{array}{c}\text { Avaliação } 1 \\
\text { (cm) }\end{array}$} & \multicolumn{2}{|c|}{$\begin{array}{c}\text { Avaliação } 2 \\
\text { (cm) }\end{array}$} & \multicolumn{2}{|c|}{$\begin{array}{l}\text { Avaliação } 3 \\
\text { (cm) }\end{array}$} & \multicolumn{2}{|c|}{$\begin{array}{c}\text { Avaliação } 4 \\
\text { (cm) }\end{array}$} & \multicolumn{2}{|c|}{$\begin{array}{c}\text { Avaliação } 5 \\
(\mathrm{~cm})\end{array}$} \\
\hline & ${ }^{3} \mathrm{PBZ}$ & & ${ }^{4} T$ & PBZ & $\mathrm{T}$ & PBZ & $\mathrm{T}$ & PBZ & $\mathrm{T}$ & PBZ & $T$ \\
\hline 1 & 64,60 & $b$ & 3,6 & 50 & 116,00 & 33 & 150 & 47,53 & $\mathrm{a}<2$ & 89,93 & $a$ \\
\hline 2 & 89,73 & 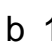 & 105, & 33 & 50 & o & 67 &, 53 & 1 & 53 & $00 \mathrm{a}$ \\
\hline 3 & 67,52 & b & 90,3 & 66,40 & b 103,00 & & 131 & 0,78 & a $1 \varepsilon$ & 0,63 & $b$ \\
\hline 4 & 69,03 & 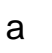 & 93,3 & ),27 & b 110,17 & & & a 127,47 & a 20 &, 80 & $a$ \\
\hline 5 & 63,60 & $b$ & 81,0 & 63,03 & b 101,33 & $37 \mathrm{~b}$ & 0 & 33 & & 3,47 & $00 \mathrm{a}$ \\
\hline 6 & 72,33 & b & $99,($ & 75,40 & b 109,67 & o & 0 &, 53 & a 19 & 1,40 & a 202,67 a \\
\hline 7 & 79,60 & b & 94 & 33 & & & & & & & 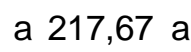 \\
\hline 8 & 77,00 & b & 84,6 & 77,73 & a 90,17 & 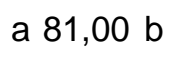 & & & a 17 & 60 & $00 \mathrm{a}$ \\
\hline 9 & 72,80 & b & 93 & & & & & & & & \\
\hline 10 & 60,63 & b & 88,6 & 62,50 & b 112,67 & a 6 & & ,33 & a 210,00 & 2,53 & a 219,00 a \\
\hline 11 & 62,40 & $b$ & 94,0 & 3,71 & b 113,50 & a & 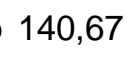 &, 56 & a 210,00 & 7,67 & $33 \mathrm{a}$ \\
\hline 12 & 76,03 & b & 96 , & 50 & & & & & a 2 & 33 & $3 a$ \\
\hline 13 & 68,47 & b & 86 & & b 89, & & & & & & $\mathrm{a}$ \\
\hline 14 & 66,40 & N & 95,0 & 3,27 & b 114,33 & 0 & 1 & 3,20 & b 21 & 47 & $\mathrm{a} 2$ \\
\hline 15 & 56,17 & b & 92,00 & 60,17 & b 120,67 & a $70,95 \mathrm{~b}$ & 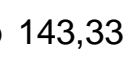 & 13 & a 188,33 &, 40 & $33 a$ \\
\hline 16 & 71,87 & b & 92,3 & 74,90 & b 111, & a & 10 & a 173,73 & a 180,00 & a 197,73 & $33 \mathrm{a}$ \\
\hline 17 & 64,01 & $b$ & 89,6 & 4,70 & b 110, & & & & a 215,00 & & $33 a$ \\
\hline 18 & 71,98 & b & 92,3 & 74,51 & b 114,33 & a 8 & 14 & 89 & a 204,67 & 0,80 & a 196,33 a \\
\hline 19 & 67,70 & & 100, & 8,50 & b 123,50 & a & 15000 & & b 225, & 60 & $b 2$ \\
\hline 20 & 60,62 & b & 85,67 & 58,83 & b 96,33 & a 65,63 b & 115,67 & a 178,13 & a 186,67 & a 169,43 & a 194,33 a \\
\hline${ }^{2} M$ & 69,13 & & 92,42 & 70,47 & 110,24 & 76,89 & 135,83 & 146,55 & 200,27 & 176,74 & 212,78 \\
\hline
\end{tabular}

As médias seguidas de mesma letra, na linha, em cada avaliação, não diferem entre si, pelo teste de Tukey, a $5 \%$ de probabilidade. ${ }^{1} \mathrm{P}$ - progênies; ${ }^{2} \mathrm{M}$ - médias; ${ }^{3} \mathrm{PBZ}$ - paclobutrazol; ${ }^{4} \mathrm{~T}$ - testemunha.

Nas avaliações 1 e 2, não houve diminuições significativas no diâmetro do caule; no entanto, observou-se tendência na diminuição do diâmetro do caule das plantas tratadas com paclobutrazol (Tabela 3). Essa tendência foi confirmada na avaliação 3 , na qual se verificou diminuição significativa do diâmetro do caule das plantas tratadas com paclobutrazol em relação às testemunhas (sem aplicação de paclobutrazol), mas essa tendência foi diminuindo ao longo das avaliações. 
Tabela 3 - Valores médios do diâmetro do caule das plantas das progênies de Eucalyptus grandis aos 12; 14; 16; 18 e 20 meses (avaliações 1, 2, 3, 4 e 5, respectivamente) sob efeito da aplicação de paclobutrazol e testemunha (sem aplicação). Averages of plants progeny diameters of Eucalyptus grandis by 12, 14, 16, 18, and 20 months (1,2, 3, 4, and 5 evaluations, respectively) under paclobutrazol effects and control (with no application).

\begin{tabular}{|c|c|c|c|c|c|c|c|c|c|c|c|c|c|}
\hline \multirow[t]{2}{*}{${ }^{1} \mathrm{P}$} & \multicolumn{3}{|c|}{$\begin{array}{l}\text { Avaliação } 1 \\
(\mathrm{~mm})\end{array}$} & \multicolumn{2}{|c|}{$\begin{array}{c}\text { Avaliação } 2 \\
(\mathrm{~mm})\end{array}$} & \multicolumn{2}{|c|}{$\begin{array}{c}\text { Avaliação } 3 \\
(\mathrm{~mm})\end{array}$} & \multicolumn{3}{|c|}{$\begin{array}{l}\text { Avaliação } 4 \\
(\mathrm{~mm})\end{array}$} & \multicolumn{3}{|c|}{$\begin{array}{l}\text { Avaliação } 5 \\
(\mathrm{~mm})\end{array}$} \\
\hline & ${ }^{3} \mathrm{PBZ}$ & & ${ }^{4} \mathrm{~T}$ & PBZ & $T$ & PBZ & $T$ & PBZ & $T$ & & PBZ & $T$ & \\
\hline 1 & 9,00 & & 3,83 & a 9,93 & a 10,84 & a 12,81 & b 19,90 & a 24,86 & a 32,67 & $\mathrm{a}$ & 29,8 & a 36,33 & \\
\hline 2 & 9,24 & a 9 &, 70 & a 10,87 & a 11,53 & a 13,53 & b 19,45 & a 24,13 & a 33,67 & a & 27,93 & a 38,33 & a \\
\hline 3 & 7,56 & a 6 &, 43 & a 8,55 & a 8,77 & a 10,64 & 18 & a 26,85 & a 30,33 & a & 30,33 & b 47,67 & $a$ \\
\hline 4 & 8,20 & a 8 & 3,52 & a 9,72 & a 10,16 & 12,13 & 33 & a 22,73 & 33 & a & 27,66 & 00 & \\
\hline 5 & 7,01 & & 7,93 & a 7,96 &, 57 & 9,31 & 31 & a 18,46 & 0,00 & a & 22,33 & a 35,00 & a \\
\hline 6 & 8,79 & a 8 & 3,63 & a 10,05 & a 11,09 & a 13,14 & b 20,33 & a 28,33 & a 31,67 & a & 31,8 & a 41,00 & a \\
\hline 7 & 9,08 & a 6 & ,73 & b 10,51 & a 8,54 & a 14,27 & 7,79 & a 29,18 & 5,00 & $\mathrm{a}$ & 34,2 &, 00 & $d$ \\
\hline 8 & 8,00 & a 6 &, 53 & 9,27 & a 7,37 & 15 & b & 20 & & $\mathrm{a}$ & 29,2 & & a \\
\hline 9 & 8,57 & a 9 & 9,30 & a 10,52 & a 11,59 & a 12,50 & b 21,07 & a 28,80 & a 36,00 & a & 33,13 & a 40,00 & a \\
\hline 10 & 7,28 & a 8 & 3,36 & a 8,96 & a 11,26 & a 11,26 & b 19,43 & a 21,33 & b 36,67 & a & 26,93 & a 38,00 & a \\
\hline 11 & 7,01 & & 7,83 & a 8,53 & a 9,81 & a 10,75 & b 23,24 & a 22,91 & b 38,67 & $\mathrm{a}$ & 27,15 & b 43,67 & $a$ \\
\hline 12 & 8,76 & a 8 & 3,77 & a 9,67 & a 10,31 & a 11,72 & b 20,47 & a 26,06 & a 34,00 & a & 32,33 & a 37,33 & a \\
\hline 13 & 9,64 & & ,05 & a 10,76 & a 11,40 & a 13,13 & b 1 & a 24,53 & a 30,67 & a & 29,53 & a 36,00 & a \\
\hline 14 & 6,98 & a 7 & 7,20 & a 8,29 & a 9,13 & a 9,86 & b 17,85 & a 21,20 & a 30,00 & a & 23,26 & a 37,00 & a \\
\hline 15 & 8,21 & a 9 & 9,03 & a 9,74 & a 12,10 & a 12,03 & b 23,22 & a 25,46 & a 31,67 & a & 32,6 & a 43,00 & a \\
\hline 16 & 8,53 & a 7 & 7,93 & a 10,00 & a 12,30 & a 13,49 & b 2 & a 27,00 & a 32,00 & $\mathrm{a}$ & 32,86 & a 35,33 & a \\
\hline 17 & 8,01 & & 9,82 & a 9,18 & b 12,35 & a 11,56 & b 19,42 & a 23,46 & a 35,67 & a & 28,4 & a 39,33 & $\mathrm{a}$ \\
\hline 18 & 8,16 & & 3,84 & a 10,60 & a 12,35 & a 14,84 & b 20,97 & a 27,86 & a 34,67 & a & 31,97 & a 39,67 & a \\
\hline 19 & 7,16 & & 7,83 & a 7,90 & a 9,54 & a 9,62 & b 15,73 & a 18,40 & b 33,50 & $\mathrm{a}$ & 20,93 & b 37,50 & a \\
\hline 20 & 5,77 & b 8 & 3,30 & a 6,60 & b 10,88 & a 8,92 & b 20,50 & a 22,50 & a 28,33 & a & 27,36 & a 36,67 & a \\
\hline$\pi$ & 05 & & 3,33 & 9,38 & 0,59 & 1,88 & 19,61 & , 41 & 2,83 & & 28, & 38,54 & \\
\hline
\end{tabular}

As médias seguidas de mesma letra, na linha, não diferem entre si, pelo teste de Tukey. ${ }^{1} \mathrm{P}$ - progênies; ${ }^{2}$ $\mathrm{M}$ - médias; ${ }^{3} \mathrm{PBZ}$ - paclobutrazol; ${ }^{4} \mathrm{~T}$ - testemunha.

Os resultados encontrados na avaliação 3 concordam com os encontrados por VARGHESE et al. (2008) em seus estudos com Eucalyptus camaldulensis e E. tereticornis. Os autores observaram redução no diâmetro do caule para plantas tratadas com o biorregulador paclobutrazol (Tabela 3).

\section{Estimativa dos parâmetros genéticos quantita- tivos}

Os resultados evidenciaram diferenças significativas $(p \leq 0,05)$ para as diferentes progênies, para as idades avaliadas, para as características altura de plantas e diâmetro do caule sob o efeito do biorregulador paclobutrazol. Portanto, com a presença de variação genética entre e dentro de progênies, possibilita-se a seleção de indivíduos mais sensíveis ou tolerantes ao biorregulador. As variâncias dentro de progênies para altura das plantas e diâmetro de caule foram, respectivamente, 798,34 e 26,66, e as variâncias genéticas entre progênies foram 191,66 e 3,42. $\mathrm{Na}$ tabela 4 abaixo, encontram-se os resultados dos parâmetros genéticos para as características altura e diâmetro do caule dos indivíduos de E. grandis presentes em pomar de polinização.

Os coeficientes de variação experimental (CV\%) para as características altura e diâmetro de caule apresentaram valores dentro dos padrões encontrados para Eucalyptus, evidenciando boa precisão experimental, variando de 8,33 a 16,18\% para as condições de vaso. Segundo PIMENTELGOMES \& GARCIA (2002), os valores para o coeficiente de variação experimental (CV\%) variam dependendo da variável de estudo, sendo que valores abaixo de $10 \%$ são baixos; 10 a $20 \%$ são médios, e acima de $30 \%$ são altos para os experimentos. 
Tabela 4 - Estimativa das herdabilidades $\left(\mathrm{h}^{2}\right)$ e dos coeficientes de variação (CV \%) aos 12; 14; 16; 18 e 20 meses sob efeito do paclobutrazol. Estimation of heritability coefficient $\left(h^{2}\right)$ and of variation coefficients (CV \%) by 12, 14, 16, 18, and 20 months old under paclobutrazol effects.

\begin{tabular}{|c|c|c|c|c|c|c|c|c|c|c|c|c|}
\hline \multirow[b]{2}{*}{${ }^{1} \mathrm{P}$} & \multicolumn{6}{|c|}{ Avaliação de Altura } & \multicolumn{6}{|c|}{ Avaliação de Diâmetro } \\
\hline & $\begin{array}{c}12 \\
\text { meses }\end{array}$ & $\begin{array}{c}14 \\
\text { meses }\end{array}$ & $\begin{array}{c}16 \\
\text { meses }\end{array}$ & $\begin{array}{c}18 \\
\text { meses }\end{array}$ & $\begin{array}{c}20 \\
\text { meses }\end{array}$ & Mé & $\begin{array}{c}12 \\
\text { meses }\end{array}$ & $\begin{array}{c}14 \\
\text { meses }\end{array}$ & $\begin{array}{c}16 \\
\text { meses }\end{array}$ & $\begin{array}{c}18 \\
\text { meses }\end{array}$ & $\begin{array}{c}20 \\
\text { meses }\end{array}$ & 1 \\
\hline${ }^{2} h^{2}{ }_{m p}$ & 0,85 & 0,84 & 0,77 & 0.64 & 0.63 & 0,75 & 0,78 & 0,66 & 0,69 & 0,52 & 0,58 & 0,64 \\
\hline${ }^{3} h^{2} d$ & 1,00 & 0,99 & 0,99 & 0,63 & 0,63 & 0,85 & 0,63 & 43 & $0,4 \varepsilon$ & 0,32 & 0,39 & 0,45 \\
\hline${ }^{4} \mathrm{~h}^{2}{ }_{\mathrm{i}}$ & 1,00 & 1,00 & 0,97 & 0,6 & 0,64 & 0,85 & 0,68 & 0,49 & 0,52 & 0,36 & 0,41 & 0,49 \\
\hline${ }^{5} \mathrm{CV}$ & 8,33 & 8,87 & 11,06 & 16,18 & 11,52 & 11,19 & 12,80 & 14,94 & 14,62 & 15,81 & 14,37 & 14,51 \\
\hline${ }^{6} \mathrm{CV}_{\mathrm{g}}$ & 11,27 & 11,67 & 11,72 & 12,45 & 12,13 & 11,78 & 13,98 & 11,99 & 12,47 & 9,48 & 9,67 & 11,52 \\
\hline${ }^{\prime} \mathrm{CV}_{\mathrm{d}}$ & 19,52 & 20,20 & 20,31 & 21,56 & 21,01 & 20,52 & 24,21 & 20,77 & 21,60 & 16,42 & 16,75 & 19,95 \\
\hline${ }^{8} \mathrm{CV}_{f}$ & 21,46 & 21,98 & 23,81 & 59,93 & 70,59 & 22,41 & 33,29 & 34,17 & 33,94 & 66,33 & 73,19 & 33,80 \\
\hline
\end{tabular}

P - parâmetros; ${ }^{2} h^{2} m p$ - herdabilidade média entre progênies; ${ }^{3} h^{2}$ - herdabilidade dentro de progênies; ${ }^{4} h_{i}^{2}$ herdabilidade individual; ${ }^{5} \mathrm{CV} \%$ - coeficiente de variação experimental; ${ }^{6} \mathrm{CV}_{\mathrm{g}}$ - coeficiente de variação genética; ${ }^{7} \mathrm{CV}_{d}$ - coeficiente de variação dentro; ${ }^{8} \mathrm{CV}_{\mathrm{f}}$ - coeficiente de variação fenotípica.

No estudo para os coeficientes de herdabilidades $\left(h^{2}{ }_{m p} ; h^{2}{ }_{d}\right.$ e $\left.h_{i}^{2}\right)$ observou-se que, para altura e diâmetro de caule, os valores foram altos nas primeiras avaliações e diminuíram ao longo do tempo, estabilizando-se a partir do $20^{\circ}$ mês sob efeito da aplicação de paclobutrazol.

Os coeficientes de herdabilidade média entre progênies $\left(\mathrm{h}^{2} \mathrm{mp}\right)$, para as características altura e diâmetro de caule, apresentaram valores médios iguais a 0,75 e 0,64 , respectivamente, mostrando elevado controle genético para as características. Os coeficientes de herdabilidade individual em nível de planta $\left(\mathrm{h}_{\mathrm{i}}^{2}\right)$ mostram que a característica com maior controle genético foi a altura de plantas $(0,85)$.

PAULA et al. (2002), estudando progênies de meios-irmãos de Eucalyptus camaldulensis encontraram valores para $\mathrm{h}^{2}$ mp iguais a 0,60 e 0,61 para altura e DAP (diâmetro à altura do peito), respectivamente. MARTINS et al. (2001), em seus estudos envolvendo progênies de meios-irmãos de Eucalyptus grandis, encontraram valores para herdabilidade média entre progênies $\left(\mathrm{h}_{\mathrm{mp}}^{2}\right)$, para altura e DAP iguais a 0,68 e 0,69, respectivamente. Estes valores são próximos aos encontrados no presente trabalho para as características altura e diâmetro de caule das plantas tratadas com paclobutrazol, em vaso.

$\mathrm{O}$ coeficiente de variação genética $\left(\mathrm{CV}_{\mathrm{g}}\right)$, para as características estudadas, permaneceu abaixo de $20 \%$, apresentando pouca oscilação durante todo o período estudado. Segundo Sebbenn et al. (1999), o coeficiente de variação genética é um parâmetro importante para os programas de melhoramento genético, por permitir um ganho adicional pela seleção dentro de progênies. Os valores das estimativas do coeficiente de variação genética dentro de progênies reforçam a hipótese da eficiência de ganhos em trabalhos de genética florestal.

ROCHA et al. (2006), estudando progê- nies de meios-irmãos de E. grandis, encontraram valores de DAP e altura iguais a $21,88 \%$ e $12,48 \%$, para o coeficiente de variação dentro $\left(\mathrm{CV}_{\mathrm{d}}\right)$, e de $25,10 \%$ e $16,26 \%$ para o coeficiente de variação fenotípica $\left(\mathrm{CV}_{\mathrm{f}}\right)$, respectivamente. Estes valores estão próximos aos encontrados neste estudo para as características altura e diâmetro de caule (Tabela 4).

Os parâmetros genéticos estimados em progênies sob o efeito de paclobutrazol, em vaso, foram próximos aos da literatura para as características altura de planta e DAP, em crescimento no campo sem os efeitos do produto paclobutrazol (PAULA et al., 2002; ROCHA et al., 2006).

\section{Conclusões}

- Paclobutrazol causou modificações na morfologia do desenvolvimento das plantas de Eucalyptus grandis, que foram intensas no início e diminuindo ao longo das avaliações.

- Os coeficientes de herdabilidade apresentaram os valores mais altos para altura de plantas e menores para diâmetro de caule, indicando, para este último, maior influência ambiental para a característica.

- Os coeficientes de variação mostraram haver maior variabilidade genética dentro do que entre progênies, para as características estudadas, apontando para maior eficiência na seleção dentro de progênies.

\section{Agradecimentos}

À Fundação de Amparo à Pesquisa do Estado de São Paulo - FAPESP, pela concessão de bolsa de estudos ao primeiro autor, processo número 04/09679-7, e à empresa Eucatex, pela doação do material genético utilizado no trabalho. 


\section{Referências}

ALMEIDA, A. C.; SOARES, J. V.; LANDSBERG, J. J.; REZENDE, G. D. Growth and water balance of Eucalyptus grandis hybrid plantations in Brazil during a rotation for pulp production. Forest Ecology and Management, Amsterdam, v.25, n.1, p.10-21, 2007.

BERBERICH, S.; SNYDER, J; GENEVE, R.; WILLIAMS, M. A. Growth and flowering response of container grown passion flower cultivars to fertilizer and paclobutrazol. Journal Environmental Horticulture, Washington, v.24, n.2, p.109-114, 2006.

CHORBADJIAN, R. A.; BONELLO, P.; HERMS, D. A. Effect of the growth regulator paclobutrazol and fertilization on defensive chemistry and herbivore resistance of Austrian Pine (Pinus nigra) and Paper Birch (Betula papyrifera). Arboriculture \& Urban Forestry, Champaign, v.37, n.6, p.278-287, 2011.

CRUZ, C. D. Programa GENES: aplicativo computacional em genética e estatística. Viçosa: UFV, 2001, 648p.

GARDNER, R. A. W.; BERTLING, I; SAVAGE, M. J. Overhead irrigation increased winter chilling and floral bud production in Eucalyptus nitens. Southern Forests, Pretoria, v.75, n.4, p.199-212, 2013.

GRIFFIN, A. R.; WHITEMAN, P.; RUDGE, T.; BURGESS, I. P.; MONCUR, M. Effect of paclobutrazol on flower-bud production and vegetative growth in two species of Eucalyptus. Canadian Journal of Forest Research, Ottawa, v.23, n.3, p.640-647, 1993.

HASAN, O.; REID, J. B. Reduction of generation time in Eucalyptus globulus. Plant Growth Regulation, Dordrecht. v.17, n.1, p.53-60, 1995.

JONES, R. C; VAILLANCOURT, R. E.; GORE, P. L.; POTTS, B. M. Genetic control of flowering time in Eucalyptus globulus ssp. globulus. Tree Genetics \& Genomes, Davis, v.7, n.6, p.12091218, 2011.

MARSHALL, J. G.; BEARDMORE, T.; WHITTLE, C. A.; WANG, B. S. P.; RUTLEDGE, R. G.; BLUMWALD, E. The effects of paclobutrazol, abscisic acid, and gibberellin on germination and early growth in silver, red, and hybrid maple. Canadian Journal of Forest Research, Ottawa, v.30, n.4, p.557-565, 2000.

MARTINS, I. S; MARTINS, R. C. C.; CORREIA, H. S. Comparação entre seleção combinada e seleção direta em Eucalyptus grandis, sob diferentes intensidades de seleção. Floresta e Ambiente, Seropédica, v.8, n.1, p.36-43, 2001.
MORAES, C. B.; ZIMBACK, L.; UESUGI, G.; GUERRINI, I. A.; MORI, E. S.; Alterações morfológicas em Eucalyptus sob a aplicação de biorreguladores. Revista do Instituto Florestal, São Paulo, v.24, n.2, p.251-257, 2012.

MORAES, C. B.; UESUGI, G.; ONO, E. O.; RODRIGUES, J. D.; GUERRINI, I. A.; MORI, E. S. Influência do uso de biorreguladores no crescimento de Hymenaea courbaril. Revista do Instituto Florestal, São Paulo, v.25, n.2, p.223229, 2013.

NETO, J. T. F.; CASTRO, A. W. V.; BIANCHETTI, A. Aplicação da seleção precoce em famílias de meios irmãos de taxi-branco. Acta Amazonica, Manaus, v.33, n.1, p.85-91, 2003.

PAULA, R. C.; PIRES, I. E.; BORGES, R. C. G.; CRUZ, C. D. Predição de ganhos genéticos em melhoramento florestal. Pesquisa Agropecuária Brasileira, Brasília, v.37, n.2, p.159-165, 2002.

PIMENTEL-GOMES, F. Curso de estatística experimental. 15.ed. Piracicaba: FEALQ, 2009. $451 \mathrm{p}$.

PIMENTEL-GOMES, F.; GARCIA, C. H. Estatística aplicada a experimentos agronômicos e florestais: exposição com exemplos e orientações para uso de aplicativos. Piracicaba: FEALQ, 2002. 309p.

QIANSHENG, L.; DENG, M.; CHEN, J.; HENNY, R. J. Effects of light intensity and paclobutrazol on growth and interior performance of Pachira aquatica Aubl. HortScience, Alexandria, v.44, n.5, p.1291-1295, 2009.

RIBEIRO, D. M.; MULLER, C.; BEDIN, J.; ROCHA, G. B.; BARROS, R. S. Effects of autoclaving on the physiological action of paclobutrazol. Agricultural Sciences, Irvine, v.2, n.3, p.191-197, 2011.

ROCHA, M. G. B.; PIRES, I. E.; ROCHA, R. B.; XAVIER, A.; CRUZ, C. D. Avaliação genética de progênies de meio-irmãos de Eucalyptus grandis por meio dos procedimentos REML/BLUP e da ANOVA. Scientia Florestalis, Piracicaba, n.71, p.99-107, 2006.

SOARES, T. S.; CARVALHO, R. M. M. A.; VALE, A. B. Avaliação econômica de um povoamento de Eucalyptus grandis destinado a multiprodutos. Revista Árvore, Viçosa, MG, v.27, n.5, p.689694, 2003.

SOUZA, C. R.; ROSSI, L. M. B.; AZEVEDO, C. P.; LIMA, R. B. Comportamento da Acacia mangium e de clones de Eucalyptus grandis x E. urophylla em plantios experimentais na Amazônia Central. Scientia Forestalis, Piracicaba, v.65, p.95-101, 2004. 
SEBBENN, A. M.; SIQUEIRA, A. C. M. F.; KAGEYAMA, P. Y.; DI DIO JUNIOR, O. J. Variação genética entre e dentro de populações de amendoim - Pterogyne nitens. Scientia Forestalis, Piracicaba, v.56, p.29-40, 1999.

STEEL, R. G. D.; TORRIE, J. W. Principles and Procedures of Statistics: a biometrical approach. $2^{\text {nd }}$ ed. New York: McGraw Hill Book Company, 1980. 633p.

TAIZ, L.; ZEIGER, E. Fisiologia vegetal. 4.ed. Porto Alegre: Editora Artmed, 2009. 848p.

VARGHESE, M.; RAVI, N.; KAMALAKANNAN, R.; HARWOOD, C. E. Effect of silvicultural treatments on growth, fertility and capsule traits in seedling seed orchards of Eucalyptus camaldulensis and E. tereticornis. New Forests, Sydney, v.37, n.1, p.99-107, 2008.
VIEIRA, S. Análise de Variância (ANOVA). São Paulo: Atlas, 2006. 204p.

WILLIAMS, D. R.; POTTS, B. M.; SMETHURST, P. J. Promotion of flowering in Eucalyptus nitens by paclobutrazol was enhancend by nitrogen fertilizer. Canadian Journal of Forest Research, Ottawa, v.33, n.1, p.74-81, 2003.

YUCEER, C.; KUBISKE, M. E.; HARKESS, R. L.; LAND JR, S. B. Effects of induction treatments on flowering in Populus deltoids. Tree Physiology, Victoria, v.23, n.7, p.489-495, 2003.

ZIMBACK, L.; MORI, E. S.; BRIZOLLA, T. F.; CHAVES, R. Correlações entre caracteres silviculturais durante o crescimento de Eucalyptus grandis Hill ex Maiden. Revista do Instituto Florestal, São Paulo, v.23, n.1, p.57-67, 2011. 